\title{
Radio Resource Allocation in LTE-Advanced Cellular Networks with M2M Communications
}

\author{
Kan Zheng*, Senior Member, IEEE, Fanglong Hu*, Wei Xiang ${ }^{\dagger}$, Senior Member, IEEE, Mischa Dohler, \\ Senior Member, IEEE ${ }^{\ddagger}$ and Wenbo Wang*, Member, IEEE \\ *Wireless Signal Processing and Network Lab \\ Key laboratory of Universal Wireless Communication, Ministry of Education \\ Beijing University of Posts \& Telecommunications \\ Beijing, China, 100088 \\ $\dagger$ Faculty of Engineering and Surveying \\ University of Southern Queensland \\ Toowoomba, QLD 4350, Australia \\ $\ddagger$ Centre Tecnologic de Telecommunications de Catalunya (CTTC) \\ Barcelona, Spain, 08860 \\ E-mail: kzheng@ieee.org
}

\begin{abstract}
Machine-to-machine (M2M) communications are expected to provide ubiquitous connectivity between machines without the need of human intervention. To support such a large number of autonomous devices, the M2M system architecture needs to be extremely power and spectrally efficient. This article thus briefly reviews the features of $\mathrm{M} 2 \mathrm{M}$ services in the third generation (3G) long-term evolution and its advancement (LTE-Advanced) networks. Architectural enhancements are then presented for supporting M2M services in LTE-Advanced cellular networks. To increase spectral efficiency, the same spectrum is expected to be utilized for human-to-human $(\mathrm{H} 2 \mathrm{H})$ communications as well as $\mathrm{M} 2 \mathrm{M}$ communications. We therefore present various radio resource allocation schemes and quantify their utility in LTE-Advanced cellular networks. System-level simulation results are provided to validate the performance effectiveness of M2M communications in LTE-Advanced cellular networks.
\end{abstract}

Index Terms- LTE-Advanced networks, M2M communications, radio resource allocation. 


\section{INTRODUCTION}

Machine-to-machine (M2M) communication is concerned with connecting communication-enabled devices in an unprecedented way, thus enabling in parts the Internet of Things (IoT) [1], [2]. With M2M communications, devices "talk" to each other through wired or wireless connections and share data without direct human intervention. The use of M2M communications is particularly well suited to interact with a large number of remote devices acting as the interface with end customers, utilities, etc. In this way, devices such as smart meters, signboards, cameras, remote sensors, laptops, and appliances can be interconnected to support a variety of new applications [3].

With the rapid development of the third generation (3G) long-term evolution cellular networks and its advancement (LTE-Advanced), M2M communications via LTE-Advanced cellular networks with widespread coverage is expected to constitute a significant part of the IoT. For mobile service operators, services through M2M communications have promising and strategic values. For instance, a large number of M2M services are non-real time and typically consume little bandwidth, with minimal impact on the capacity of radio access networks (RANs). With M2M services supported by information and communication technologies (ICT), operators can expand their end-to-end information solutions into industries beyond the currently supported.

Unlike traditional human-to-human $(\mathrm{H} 2 \mathrm{H})$ services, such as voice and web streaming, M2M services often have very different requirements on a communication system due to their specific features [4]. Another distinguishing characteristic in cellular networks with $\mathrm{M} 2 \mathrm{M}$ communication is the large increase in the number of machine-type communication (MTC) devices. Both of them bring forth new challenges for LTE-Advanced cellular networks, demanding significant improvements in the efficiency of radio resource utilization. Meanwhile, new M2M services have to have little or even no impact on existing $\mathrm{H} 2 \mathrm{H}$ services in cellular networks [5]. Preliminary studies on M2M communications have primarily thus focused on service requirements, the functional architecture and applications [4], [6].

Concerning the service requirements, $\mathrm{M} 2 \mathrm{M}$ applications are quite different from their $\mathrm{H} 2 \mathrm{H}$ counterparts since M2M services have their own very unique characteristics [4], [7]. Moreover, Quality-of-Service (QoS) requirements of different types of M2M services vary widely and are reflected in the MTC service 
features: group-based communications, low or no mobility, time-controlled, time-tolerant, small data transmission, secure connection, MTC monitoring, priority alarm messages, extra low power consumption, etc. These service requirements then dictate the architectural design, to be discussed below.

With an architecture in place, numerous challenges remain for radio resource management (RRM) for M2M communication in LTE-Advanced cellular networks. For example, time and frequency resources are to be shared between $\mathrm{H} 2 \mathrm{H}$ users and MTC devices (MTCDs), thus resulting in co-channel interference among them [8]. Such co-channel interference plays a detrimental role in degrading the performance of the LTE-Advanced cellular networks with M2M communications. Furthermore, differentiated QoS requirements between $\mathrm{H} 2 \mathrm{H}$ users and MTCDs have to be accommodated, which requires different interference tolerances for different types of users and devices. To the best of the authors' knowledge, there has been no work in the literature on radio resource allocation for LTE-Advanced cellular networks with M2M communications so far.

The scope of this article is hence to examine how $\mathrm{H} 2 \mathrm{H}$ users and MTC devices can share available radio resources efficiently so as to mitigate co-channel interference and thus enhance network efficiency. We first introduce some architectural enhancements needed to fulfill above MTC service requirements. Then, several radio resource allocation schemes are proposed for LTE-Advanced cellular networks with M2M communications. We then analyze and assess their performance extensively through system-level simulations and study $\mathrm{H} 2 \mathrm{H}-\mathrm{M} 2 \mathrm{M}$ coexistence issues.

\section{Architectural Enhancements to Fulfill M2M Service ReQuirements}

In order to meet the requirements of LTE-Advanced such as peak data rates of up to $1 \mathrm{Gbit} / \mathrm{s}$, more spectrum bands are needed. Besides the existing carriers for 3G networks, spectrum bands located at 450-470 MHz, 698-790 MHz, 2.3-2.4 GHz and 3.4-3.6 GHz can be used for the deployment of LTE and LTE-Advanced networks [9]. Moreover, LTE-Advanced has been defined to support scalable carrier bandwidth exceeding $20 \mathrm{MHz}$, potentially up to $100 \mathrm{MHz}$, in a variety of carriers for deployments.

The current RAN for LTE-Advanced consists of a single node, i.e., the eNodeB (eNB) that provides the user plane and control plane protocol terminations towards the user equipment (UE). It is a fully distributed radio access network architecture, where eNBs may be interconnected with each other by 
means of the X2 interface. Meanwhile, eNBs are connected through the S1 interface to the core network. In each eNB, there exist the PHYsical (PHY), Medium Access Control (MAC), Radio Link Control (RLC), and Packet Data Control Protocol (PDCP) layers that implement the functionality of user-plane header-compression and encryption. The current 3G LTE cellular network is designed only for providing $\mathrm{H} 2 \mathrm{H}$ services for user equipments (UEs). However, with the introduction of M2M communications, the network architecture needs to be improved to accommodate M2M service requirements without sacrificing the qualities of current $\mathrm{H} 2 \mathrm{H}$ services.

\section{A. MTCD-Related Communications}

To enable M2M communications, two new network elements, i.e., the MTCD and MTC gateway (MTCG), appear in LTE-Advanced cellular networks. A MTCD is a user equipment (UE) designed for machine-type communications, which communicates through a cellular network with an MTC server and/or other MTCDs. The network requires an MTCG gateway to facilitate communications among a great many MTCDs and to provide a connection to a backhaul that reaches the Internet. The MTCG will be able to intelligently manage power consumptions of the network, and provide an efficient path for communications between MTCDs. Three different M2M communications methods are feasible, as illustrated in Fig. 1

1) Direct transmission between MTCD and eNB: Similar to a normal UE, an MTCD has the ability to establish a direct link with its donor eNB. Therefore, there exist strong similarities between the eNBto-UE and eNB-to-MTCD links. On the other hand, MTCDs normally appear in large quantities in the M2M networks and thus exhibit the service feature of group-based communications. In certain time instants, intense competition for radio resources may occur. For instance, one or more MTC groups send communication requests to an eNB simultaneously, which may cause network congestion, resulting in performance degradation for both $\mathrm{M} 2 \mathrm{M}$ and $\mathrm{H} 2 \mathrm{H}$ services. Therefore, additional efforts have to be made to tackle such kinds of problems, when a large quantity of MTCDs communicate with the eNB directly.

2) Multi-hop transmission with the aid of an MTC gateway: In order to mitigate or eliminate negative effects of M2M communications on $\mathrm{H} 2 \mathrm{H}$ communications, an MTC gateway can be deployed in celluar networks, where all MTCDs are connected to the eNB indirectly through the relaying of the MTCG. In 
other words, the end-to-end communication between the eNB and MTCDs may occur via more than one hop, e.g., the eNB-to-MTCG and MTCG-to-MTCD links. Besides, MTCDs may establish peer-to-peer communications with each other with the aid of the MTCG or eNB. The eNB-to-MTCG wireless link is based on 3G LTE specifications, whereas the MTCG-to-MTCD and MTCD-to-MTCD communications can either be via 3G LTE specifications or other wireless communications protocols such as IEEE 802.15.x. The resulting multi-level network management problem can be handled with the aid of the MTCG. Each MTCD is controlled by its donor MTCG, which is managed by the eNB. The introduction of the MTCG makes the network topology more complex, leading to challenges as well as opportunities.

MTCDs are usually grouped for control, management or charging facilities. The MTCDs within the same group can be in the same area and/or possess the same MTC features. Each MTCG can serve one or more groups.

3) Peer-to-peer transmission between MTCDs: An MTCD may communicate locally with other entities, which provide the MTCD with raw data for processing and communicating to the MTC server and/or other MTCDs. Compared to other local connectivity solutions, such as IEEE 802.11a or IEEE 802.15.x, peer-to-peer transmission between MTCDs supported by a cellular network offers appealing advantages. The cellular network can broadcast local services available within a much wider coverage area. Thus, for automated service discovery, the MTCDs do not have to constantly scan for available local access points (APs) as in case of IEEE 802.11a. This is advantageous since leading to significantly reduced power consumption for scanning. With the knowledge of encryption keys at both MTCDs involved in peer-to-peer communications, a secure connection can be established without manual pairing of devices or entering encryption keys. Moreover, through the control of the eNB via peer-to-peer communications, the interference to other cellular receivers can be limited or mitigated.

\section{B. Architectural Enhancements}

In order to support $\mathrm{M} 2 \mathrm{M}$ communications, the RAN architecture needs to be enhanced to enable coexisting communications between MTCD-related and $\mathrm{H} 2 \mathrm{H}$ communications in LTE-Advanced cellular networks. Fig. 2 gives an example of architectural enhancements to the M2M cellular network.

Apart from direct transmission, MTCDs can also establish communications with their donor eNBs 
through multi-hop transmission. To avoid self-interference and reduce implementation complexity, halfduplex MTCGs are preferred for deployment in the networks. Furthermore, when local services are available between nearby MTCDs, peer-to-peer communications provided by cellular networks may appear to be a local connectivity solution.

In such fairly intricate M2M cellular networks, how to assign and coordinate radio resources to different classes of transmissions becomes a critical issue, which will be dealt with in the next section.

\section{RADIO RESOURCE ALLOCATION FOR M2M COMMUNICATIONS}

Introducing M2M communications to LTE-Advanced cellular networks should not come at the expense of significantly degraded performance for existing $\mathrm{H} 2 \mathrm{H}$ communications. There are two major methods for radio resource allocation between $\mathrm{M} 2 \mathrm{M}$ and $\mathrm{H} 2 \mathrm{H}$ communications, i.e., orthogonal and shared resource allocation. Collocating M2M and $\mathrm{H} 2 \mathrm{H}$ communications in orthogonal channels is a simple solution but leads to low spectral efficiency from a system level perspective. To achieve higher spectral efficiency, $\mathrm{M} 2 \mathrm{M}$ communications can reuse the radio resources assigned to $\mathrm{H} 2 \mathrm{H}$ communications, resulting in shared channel allocation. However, this will cause an increased level of interference in comparison with orthogonal channel allocation.

In LTE-Advanced networks, radio resources are usually divided into resource blocks (RBs) along the frequency domain per time slot, which is also referred to as subchannels in radio resource allocation. In mixed $\mathrm{H} 2 \mathrm{H}$ and $\mathrm{M} 2 \mathrm{M}$ communications networks, there are usually five types of links as illustrated in Fig. 3(a), namely, 1) the eNB-to-UE link; 2) the eNB-to-MTCD link; 3) the eNB-to-MTCG link; 4) the MTCG-to-MTCD link; and 5) the MTCD-to-MTCD link. When radio resources are shared among these links, interference becomes a challenging issue. Therefore, it is essential to first design the efficient radio resource partition in such networks.

The radio resource partition aims at applying restrictions to the radio resource management in a coordinated way among nodes. These restrictions can be either on the available radio resources or in the form of restrictions on the transmit power that can be applied to certain radio resources. Such restrictions provide the possibility for improvement in Signal-to-Interference-plus-Noise ratio (SINR), and consequently to the cell edge performance and coverage. It is critical to exploit the characteristics 
of links to obtain the well-designed coordination pattern, which can achieve performance gain for almost all users in the network. Fig. 3(b) shows an example of radio resource partition pattern for the downlink transmission of LTE-Advanced cellular networks with M2M communications. On the assumption of halfduplex MTCGs, every two time slots are grouped together as one basic unit for transmission. In the first slot, termed as the backhaul slot, the MTCGs receive signals from the eNB. In the second slot, called the access slot, MTCGs send the data to their serving MTCDs.

In the backhaul slot, the eNB-to-MTCG transmission link has to be reliable to ensure the service quality of the MTCDs associated with the MTCG. Thus, the eNB-to-MTCG links are assigned orthogonal parts of the radio resources, whereas all other links directly associated with the eNB share the channel. In the access slot, all links except for the eNB-to-MTCG links share all the radio resources using various methods.

MTCD-to-MTCD communications are envisaged to take place only locally with relatively low power, and using either uplink or downlink channel. This implies that these links do not interfere with any other links, whereas the inverse of course does not hold true. The MTCG-to-MTCD link, however, can strictly generate interference with ongoing communication links. However, since these devices typically serve some spatially very small areas where coverage is typically poor, the impact onto the other links is neglected here. Incorporating a complete interference scenario is possible but unlikely to change the design insides.

With the resource partition pattern given in Fig. 3(b), not all the interferences between different links have to be specifically dealt with. So, we only focus on resource allocation between some of links in the following parts.

\section{A. Orthogonal Allocation for the eNB-to-MTCG Link}

Radio resource allocation and scheduling between the MTCG and MTCDs can be carried out at the MTCG in coordination with its donor eNB. Instead of communicating with an eNB directly, the MTCDs associated with a MTCG first establish a link with the MTCG. Via such multi-hop transmission, intense competition against radio resources can be mitigated especially when enormous MTCDs request access to the network resources simultaneously. In addition, the radio resources can also be reused between MTCGs 
in the case of multiple MTCGs per cell to improve on the spectral efficiency of the network.

As mentioned before, there is no co-channel interference between the eNB-to-MTCG link and other links due to orthogonal channel allocation in the backhaul slot. For the purpose of achieving high spectral efficiency, resource allocation for the eNB-to-MTCG link needs to be adjusted semi-statically according to service demands from the MTCDs associated with the MTCG. If there are not enough resources available for data transmission between the eNB and MTCG, the associated MTCDs can not be served in time, resulting in QoS degradation. Otherwise, when excessive resources are assigned to the eNB-to-MTCG link, the QoS performance of other users such as UEs and other MTCDs may suffer due to insufficient radio resources. Hence, resource partition in the backhaul slot is rather crucial to the overall system performance.

Fortunately, MTCDs usually possess common service features of small data transmission and timetolerance, which implies that the average or maximum data rate of MTCDs can be easily known according to the service type or device type. With the knowledge of M2M services, the eNB can first roughly estimate the total data rate of all MTCDs attached to a MTCG. Then, the number of RBs needed for the transmission between the eNB and MTCGs in the backhaul slot can approximately be calculated through dividing the total data rate by the average data rate per RB in the eNB-to-MTCG link.

\section{B. Scheduling Between the eNB-to-UE and eNB-to-MTCD Links}

For LTE-Advanced cellular networks with both $\mathrm{H} 2 \mathrm{H}$ and $\mathrm{M} 2 \mathrm{M}$ services, the user utility of a service is more informative than the simple QoS indicator due to the diversity of the applications. Generally speaking, the user utility of a service is a measurement of its QoS performance based on the provided network services such as the bandwidth, transmission delay and loss ratio. It describes the satisfaction level of the service delivered to the application. Here we focus on the user utility as a function of the achievable data rate only, which is most commonly used in the literature. With the control of the eNB, radio resources can be efficiently shared between the eNB-to-UE and eNB-to-MTCD links by using the utility-based scheduling scheme.

We can classify network applications into four classes and their features are shown as follows [11]: 
- Class 1 (Elastic Applications): Such applications are rather tolerant of delays. Traditional H2H data applications like file transfer, electronic mail are typical ones of this kind. Another example is file downloading of remote MTCDs from MTC servers. Their user utility has diminishing marginal improvements with incremental increase in the achievable data rate, and can be described as a strict concave function.

- Class 2 (Hard Real-Time Applications): These applications need their data to be served within a given delay constraint. Otherwise, there is no extra utility gain even with further increase of the data rate. An example of such applications is traditional telephony. For applications with hard real-time requirements, the user utility is a step function of the achievable data rate. Vehicle and asset tracking, a typical M2M service application, has to monitor and manage the MTCDs in real-time, which is also a hard real-time application.

- Class 3 (Delay-Adaptive Applications): Applications like audio and video services are delay sensitive. However, most of these applications can be made rather tolerant of occasional delay-bound violation and dropped packets. They have an intrinsic data rate requirement, and the user utility deteriorates rapidly only when the achievable data rate is below the requirement. There are a great number of such applications in M2M communications, e.g., remote monitoring in e-Health services.

- Class 4 (Rate-Adaptive Applications): Rate-adaptive applications adjust their transmission rates according to available radio resources while maintaining moderate delays. Thus, the performance of these applications highly depends on the scheduling scheme and the quality of the underlying wireless channel. Obviously, the increase of utility with the increase of the data rate is only marginal at high data rates. Conversely, the increase of utility will not be significant at very low data rates owing to the unbearably low signal quality.

Fig. 4 illustrates four example utility functions corresponding to the four classes discussed above. Due to different requirements of the applications, it is likely that $\mathrm{H} 2 \mathrm{H}$ and $\mathrm{M} 2 \mathrm{M}$ services have various formats or parameters for their utility functions, even though they fall under the same class.

Usually the resource allocation problems for UEs and MTCDs can be solved with different objectives. Consider a network with a UE set $\mathcal{H}$ and a MTCD set $\mathcal{M}$, with each UE or MTCD having its own utility function depending on the specific application. When the objective of maximizing the aggregate utility 
(MAX-Utility) is assumed as an example, radio resource allocation for the eNB-to-UE and eNB-to-MTCD links can be formulated as

$$
S^{*}=\arg \max _{S \in \mathcal{S}}\left\{\sum_{i \in \mathcal{H}} U_{i}^{H}\left(R_{i}^{H}\right)+\lambda \sum_{j \in \mathcal{M}} U_{j}^{M}\left(R_{j}^{M}\right)\right\}
$$

where $S \in \mathcal{S}$ represents a possible resource allocation matrix, $R_{i}^{H}$ and $R_{j}^{M}$ represent the achievable data rate of the $i$ th UE and $j$ th MTCD, respectively, $U_{i}^{H}\left(R_{i}^{H}\right)$ and $U_{j}^{M}\left(R_{j}^{M}\right)$ are the corresponding utility functions, and $\lambda \in[0,1]$ is the unified weighting factor of $\mathrm{M} 2 \mathrm{M}$ communication. All radio resources are orthogonally allocated to users using the utility-based scheduling scheme according to their pre-defined utility functions. In lieu of the data rate, the utility has become the metric used in resource allocation. When only slight or no utility improvement is achieved with the increase of the data rate, users will not be assigned radio resources.

\section{Allocation between MTCD-to-MTCD Links}

In order to improve network efficiency, it is assumed that different MTCD-to-MTCD links share the same radio resources. Moreover, MTCD-to-MTCD transmission can share the resources used by the other links owing to the low transmission power of MTCDs. In general, the assignment of subchannels between MTCD-to-MTCD links can be performed through the centralized or distributed way. The former can achieve a higher resource efficiency with much more overhead and complexity than the latter.

For the sake of implementation, a distributed graph-based approach can be applied for the channel assignment for MTCD-to-MTCD links. In an interference graph, each vertex denotes a pair of active MTCDs and an edge represents the interference condition between two pairs. The edge exists only when the channel gain difference between the interfering and serving links exceeds a certain threshold. One color represents a subchannel. By measuring the reference signal transmitted by its neighboring MTCDs, an MTCD is able to know the device identification of each neighboring MTCD and the pathloss between itself and its neighboring $\mathrm{MTCD}(\mathrm{s})$. Then, each MTCD has the information of its own local interference graph and negotiates with other MTCDs. MTCDs can request/release some subchannels to improve on the system utility while complying with conflict constraints imposed by other neighbors.

Each vertex, i.e., an MTCD pair, is responsible for assigning its own color, i.e., subchannel. After randomly choosing an initial color, the vertex repeatedly chooses a color that minimizes the number 
of conflicts it has with its neighbors based upon the knowledge of its neighbors' colors. Each vertex simultaneously chooses a color for itself. When a vertex changes the color, it also communicates its new color to its neighbors in time. The decision to use more than one subchannel at a vertex is probabilistic. Each vertex determines an activation probability, dependent on its degree and resources already occupied. Then, the vertex generates a random number in the range of $[0,1]$, and decides to activate one more subchannel if the random number falls below its activation probability. The method for determining the activation probability can significantly impact on the performance.

\section{Performance Evaluation}

In this section, the performances of LTE-A cellular networks with M2M communications under the urban scenarios are evaluated through system-level simulations. The detailed simulation parameters including the channel model and system configurations are summarized in Table [1 [12], which mostly are defined in 3rd Generation Partnership Project (3GPP) specifications. All UEs are evenly distributed in the circular areas around each eNB. The MTCD placement is performed as follows: 50 MTCDs are located uniformly per sector while 50 pairs of MTCDs are deployed uniformly at random in a floor of a building, all duty cycled at $10 \%$. To simulate the realistic scenarios where mixed $\mathrm{H} 2 \mathrm{H}$ and $\mathrm{M} 2 \mathrm{M}$ services exist in the cellular network, different utility functions are assumed for the UEs and MTCDs, respectively [13], i.e., Class 1 for UEs while Class 4 for MTCDs.

When M2M communications are introduced into the network, the performances of existing $\mathrm{H} 2 \mathrm{H}$ communications are somehow affected due to the decrease of the available radio resources. When the Max-Utility scheduling scheme is applied, such effects can be controlled by adjusting the unified weighting factor of M2M communications, i.e., $\lambda$. In Fig. 5(a), we compare the user utility performances in terms of the given percent point of the cumulative distribution function (CDF) in the networks with different values of $\lambda$. With the increase of the factor value, the performance of M2M communication is improved while the cell edge user performance, i.e., the $10 \% \mathrm{CDF} \mathrm{H} 2 \mathrm{H}$ performance, deteriorates. Such improvement in $\mathrm{M} 2 \mathrm{M}$ communication and degradation in $\mathrm{H} 2 \mathrm{H}$ communication levels off when $\lambda$ is larger than 0.8 . On the other hand, the performances of $\mathrm{H} 2 \mathrm{H}$ communication located in the cell center, i.e., $50 \%$ and $90 \% \mathrm{CDF} \mathrm{H} 2 \mathrm{H}$ performances, remain virtually unchanged with a variation of $\lambda$. Therefore, $\lambda=0.8$ is used for the network with mixed $\mathrm{H} 2 \mathrm{H}$ and M2M services. Then, Fig. 5(b) presents the CDF performance 
TABLE I

PARAMETER CONFIGURATIONS IN LTE-ADVANCED CELLULAR NETWORKS

\begin{tabular}{|c|c|c|}
\hline \multicolumn{2}{|l|}{ Parameter } & Values \\
\hline \multicolumn{2}{|c|}{ Cellular layout } & 19 cells / 3 sectors per cell \\
\hline \multicolumn{2}{|c|}{ Inter-site distance (ISD) } & $500 \mathrm{~m}$ \\
\hline \multicolumn{2}{|c|}{ Macro UE density } & 5 UEs / sector \\
\hline \multicolumn{2}{|c|}{ MTCD placement } & $\begin{array}{l}5 \text { MTCDs per sector, } \\
10 \text { pairs of MTCDs in apartments }\end{array}$ \\
\hline \multicolumn{2}{|c|}{ Macro cell shadowing standard deviation } & $8 \mathrm{~dB}$ \\
\hline \multirow[t]{2}{*}{ Macro cell shadowing correlation } & Between cells & 0.5 \\
\hline & Between sectors & 1 \\
\hline \multicolumn{2}{|c|}{ Max eNB transmit power } & $46 \mathrm{dBm}$ \\
\hline \multicolumn{2}{|c|}{ eNB antenna gain after cable loss } & $14 \mathrm{dBi}$ \\
\hline \multicolumn{2}{|c|}{ Max MTCD transmit power } & $14 \mathrm{dBm}$ \\
\hline \multicolumn{2}{|c|}{ UE antenna gain } & $0 \mathrm{dBi}$ \\
\hline \multicolumn{2}{|l|}{ Noise figure } & $9 \mathrm{~dB}$ \\
\hline \multicolumn{2}{|c|}{ Apartment block } & $\begin{array}{c}\text { Two stripes with } \\
1 \times 4 \times 10 \text { (floor } \times \text { row } \times \text { column) for each stripe }\end{array}$ \\
\hline \multicolumn{2}{|c|}{ Number of blocks per cell } & 1 \\
\hline \multirow[t]{2}{*}{ Pathloss } & eNB-to-UE/MTCD & $128.1+37.6 \log (R)$ in $\mathrm{dB}, R$ in $k m$ \\
\hline & MTCD-to-MTCD & $\begin{array}{c}\text { LOS: } 38.5+20 \log (R), R<0.3, R \text { in } m \\
\quad \text { NLOS: } 48.9+40 \log (R), R \geq 0.3\end{array}$ \\
\hline
\end{tabular}

of the user utility with and without concurrent M2M communications. It is observed clearly that the performance of UEs at the cell edge is degraded when MTCDs are introduced to the network. This is due to the parameter setting of the utility functions for $\mathrm{M} 2 \mathrm{M}$ and $\mathrm{H} 2 \mathrm{H}$ communications in our simulations. The utility of MTCDs increases more rapidly than that of the UEs in the low rate region. Then, MTCDs rather than UEs are more likely selected by the Max-Utility scheduling scheme when the achievable rate is not high. In other words, we can adjust the scheduling priority of $\mathrm{M} 2 \mathrm{M}$ and $\mathrm{H} 2 \mathrm{H}$ communications in the given data region by applying the specific formats of the utility functions. On the other hand, besides the existing $\mathrm{H} 2 \mathrm{H}$ communications, MTCDs with simultaneous M2M communications contribute to the aggregated cell utility. The utility achieved by all MTCDs is larger than the utility degradation of the cell edge UEs. Hence, the performance of the aggregated cell utility is improved, i.e., 4.0264 with and 2.8714 without M2M communications, respectively. It is noted that such a gain depends on several factors including the parameters of the utility functions, the number of MTCDs, unified weighting factor of M2M communications, etc.

As shown in Fig. 6, we examine the user utility performance of MTCDs deployed in urban buildings with peer-to-peer transmission, where the full reuse and graph-based channel allocation approaches are 
applied, respectively. When the graph-based approach is applied to deal with radio resource allocation between MTCDs, the interference between the different links is well controlled. Then, compared with the full reuse approach, the received SINRs of MTCDs are increased especially at the cell edge. Consequently, the utility performance is improved, e.g., in $90 \%$ operational cases, the full reuse approach achieves a utility of only 0.15 , whereas the graph-based approach yields at least 0.5 . Moreover, the gain in the low or medium data rate region is more obvious than that in the high data rate region. This is because the utility of MTCDs is a non-linear function of the data rate per resource block, i.e., the utility increases much more rapidly in the low or medium data rate region than in the high data rate region.

\section{CONCLUSiOnS}

M2M communications are clearly an emerging technology and a facilitator of the IoT by means of, among others, cellular technology. It has thus gained increasing attention in LTE-Advanced cellular network designs. In this paper, we first presented the required network architectural enhancements with the introduction of various transmission schemes related to MTCDs. Then, several radio resource allocation schemes for different transmission links have been proposed with the aim of minimizing co-channel interference and maximizing network efficiency. Our simulation results demonstrate that the proposed schemes can improve the network performance in terms of user utility.

In the next step, practical issues will be paid more attention to when designing new resource allocation schemes for M2M communications. Firstly, typical application scenarios and M2M service features will be kept in line with the development of the standardization bodied such as 3GPP and ETSI M2M. Then, to meet the specified requirements of given scenarios and services, more types of schemes are developed with different objectives. Moreover, the overhead and complexity for implementing the schemes have to be considered in order to strike a right balance between performance and cost. It is expected that the well-designed resource allocation schemes can bring to operators remarkable benefits at affordable costs in LTE-Advanced M2M-enabled cellular networks.

\section{ACKNOWLEDGMENT}

This work was supported in part by ICT project INFSO-ICT-258512 EXALTED, National Key Technology R\&D Program of China under Grant 2009ZX03003-008-01 and Research Fund for the Doctoral 
Program of Higher Education under Grant 20090005120002.

\section{REFERENCES}

[1] Commission of the European Communities, "Internet of Things - An action plan for Europe," June, 2009.

[2] A. Luigi, I. Antonio, M. Giacomo, "The Internet of Things: A survey," International Journal of Computer and Telecommunications Networking, vol. 54, no. 15, pp. 2787-2805 October, 2010.

[3] V. Gonclves, P. Dobbelaere, "Business Scenarios for Machine-to-Machine Mobile Applications," Proc. International Conference on Mobile Business and Global Mobility Roundtable (ICMB-GMR), pp.394-401, 2010.

[4] 3GPP TS 22.368 v1.0, "Service requirements for Machine-Type Communications (MTC) Stage 1 (Release 10)," March, 2010.

[5] 3GPP TR 23.888 v0.5.1, "System Improvements for Machine-Type Communications (Release 10)," July,n2010.

[6] ETSI TS 102 689, v1.1.1, "Machine-to-Machine communications (M2M): M2M service requirements," August, 2010.

[7] M. Dohler, T. Watteyne, J. Alonso, "Machine-to-Machine: An Emerging Communication Paradigm," Tutorial, PIMRC 2010, 26 September 2010, Istanbul, Turkey; also at Globecom 2010, Miami 2010, USA.

[8] K. Doppler, M. Rinne, C. Wijting, C. Ribeiro, K. Hugl,, "Device-to-device communication as an underlay to LTE-advanced networks," IEEE Communications Magazine, vol. 47, no. 12, pp. 42-49, 2009.

[9] Framework and Overall Objectives of the Future Development of IMT- 2000 and Systems Beyond IMT-2000, Recommendation ITU-R M.1645, June 2003.

[10] IST WINNER D3.5.1, "Relaying concepts and supporting actions in the context of CGs," Online: http://www.ist-winner.org/WINNER2-Deliverables/D3.5.1_v1.0.pdf.

[11] S. Shenker, "Fundamental design issues for the future Internet," IEEE Selected Areas in Communications, vol.13, no.7, pp. 1176-1188, Sep. 1995.

[12] 3GPP TR 36.814, v2.0.0, "Further Advancements for E-UTRA, Physical Layer Aspects," March, 2010.

[13] J. W. Lee, R. R. Mazumdar, N. B. Shroff, "Non-Convex Optimization and Rate Control for Multi-Class Services in the Internet," IEEE/ACM Transactions on Networking, vol. 13, no. 4, pp. 827-840, Aug. 2005.

\section{BIOGRAPHY}

Kan Zheng (M'03, SM'09) received the B.S., M.S. and Ph.D degree from Beijing University of Posts\&Telecommunications (BUPT), China, in 1996, 2000 and 2005, respectively, where he is currently associate professor. He worked as a senior researcher in the companies including Siemens, Orange Labs R\&D (Beijing), China. His current research interests lie in the field of machine-to-machine (M2M) communication, cooperative communication and heterogeneous networks.

Fanglong Hu received the B.S. degree from Beijing University of Posts\&Telecommunications (BUPT), China, in 2009. Since then he has been working toward a M.S. degree in BUPT. His research interests include machine-to-machine (M2M) communication and heterogeneous networks.

Wei Xiang (M'04, SM'10) received the B.Eng. and M.Eng. degrees, both in electronic engineering, from the University of Electronic Science and Technology of China, Chengdu, China, in 1997 and 2000, respectively, and the Ph.D. degree in telecommunications engineering from the University of South Australia, Adelaide, Australia, in 2004. Since January 2004, he has been with the Faculty of Engineering and Surveying, University of Southern Queensland, Toowoomba, Australia, where he was first an Associate Lecturer in Computer Systems Engineering from 2004 to 2006, then a Lecturer from 2007 to 2008, and currently holds a faculty post of Senior Lecturer. His research interests are in the broad area of communications and information theory, particularly coding and signal processing for multimedia communications systems. He was a visiting scholar to Nanyang Technological University from January to June 2008, and the University of Mississippi from October 2010 to March 2011, respectively. He received a prestigous Queensland International Fellowship awarded by the State Government of Queensland, Commonwealth of Australia, in 2010.

Mischa Dohler (mischa.dohler@cttc.es) is now leading Intelligent Energy [IQe] at CTTC in Barcelona, as well as being CTO of Worldsensing. Prior to this, he was at France Telecom and Kings College London. He is working on smart grid, machine-to-machine, femto, cooperative, cognitive and docitive networks. He has published several books and more than 130 refereed papers, holds several patents, has given numerous tutorials, and participated in standardization activities. He has been chair and TPC of various conferences. He is and has been editor for numerous IEEE and non-IEEE journals, as well as being the EiC of ETT. He is Senior Member of the IEEE and fluent in 6 languages.

Wenbo Wang (M'98) received his B.S., M.S. and Ph.D degree from Beijing University of Posts\&Telecommunications (BUPT), China, in 1986, 1989 and 1992 respectively. He is currently a professor and dean of graduate school in BUPT. His research interests include signal processing, mobile communications and wireless network. 


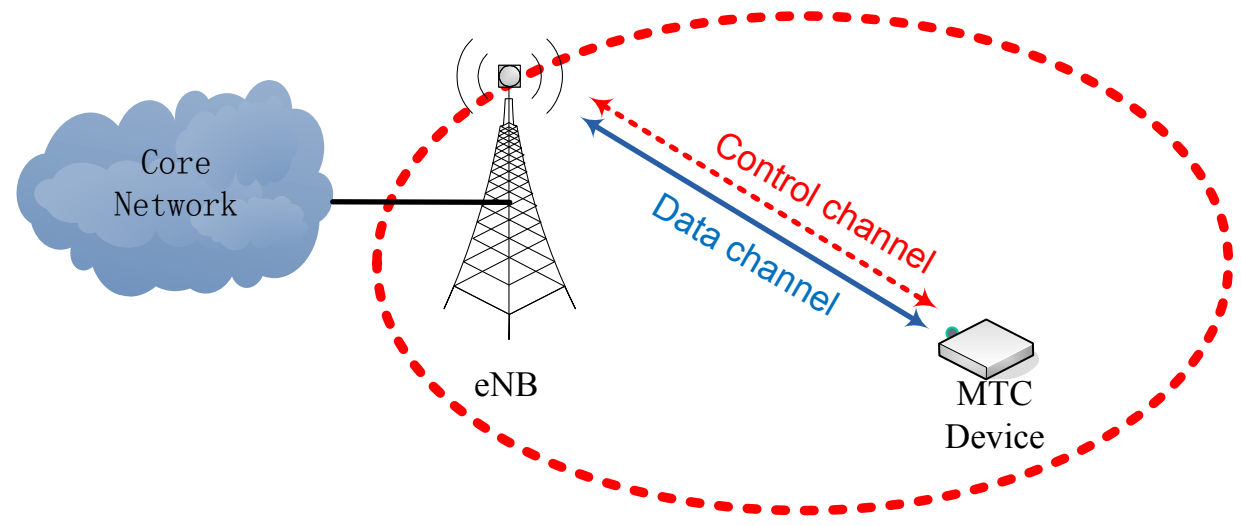

(a) Direct transmission.

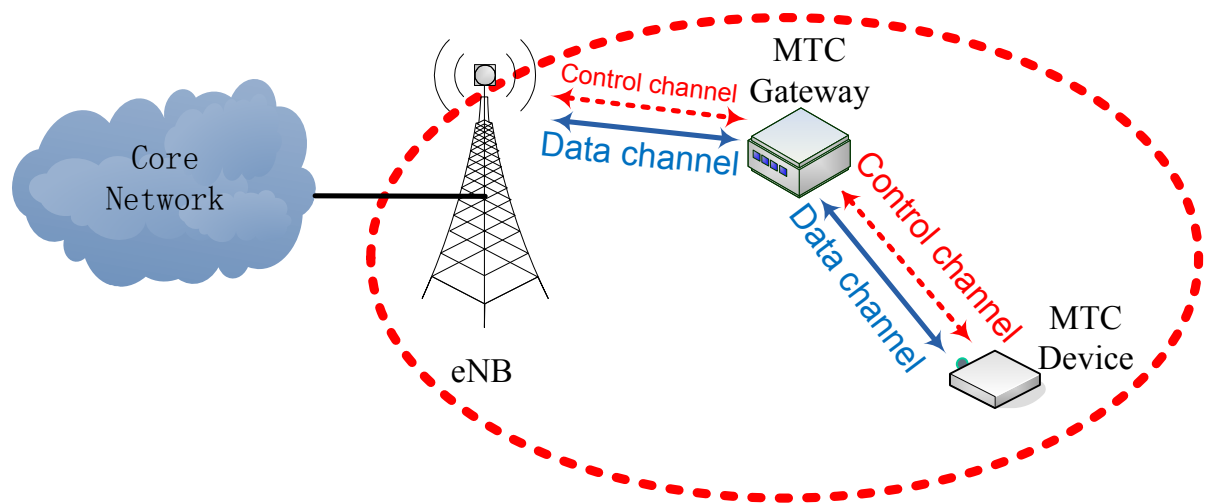

(b) Multi-hop transmission.

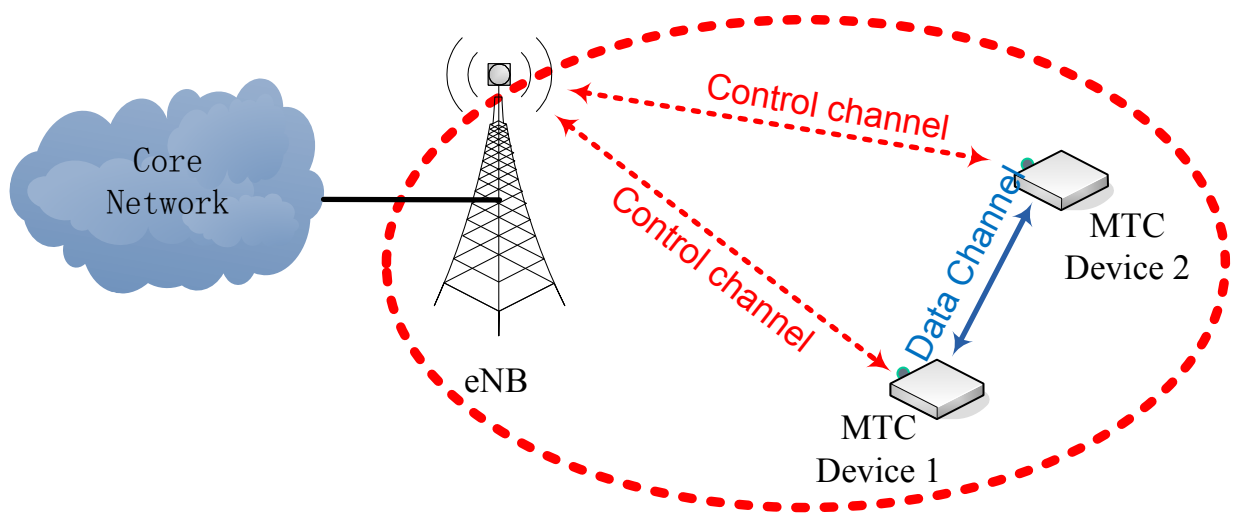

(c) Peer-to-peer transmission.

Fig. 1. Illustration of MTCD-related transmission. 


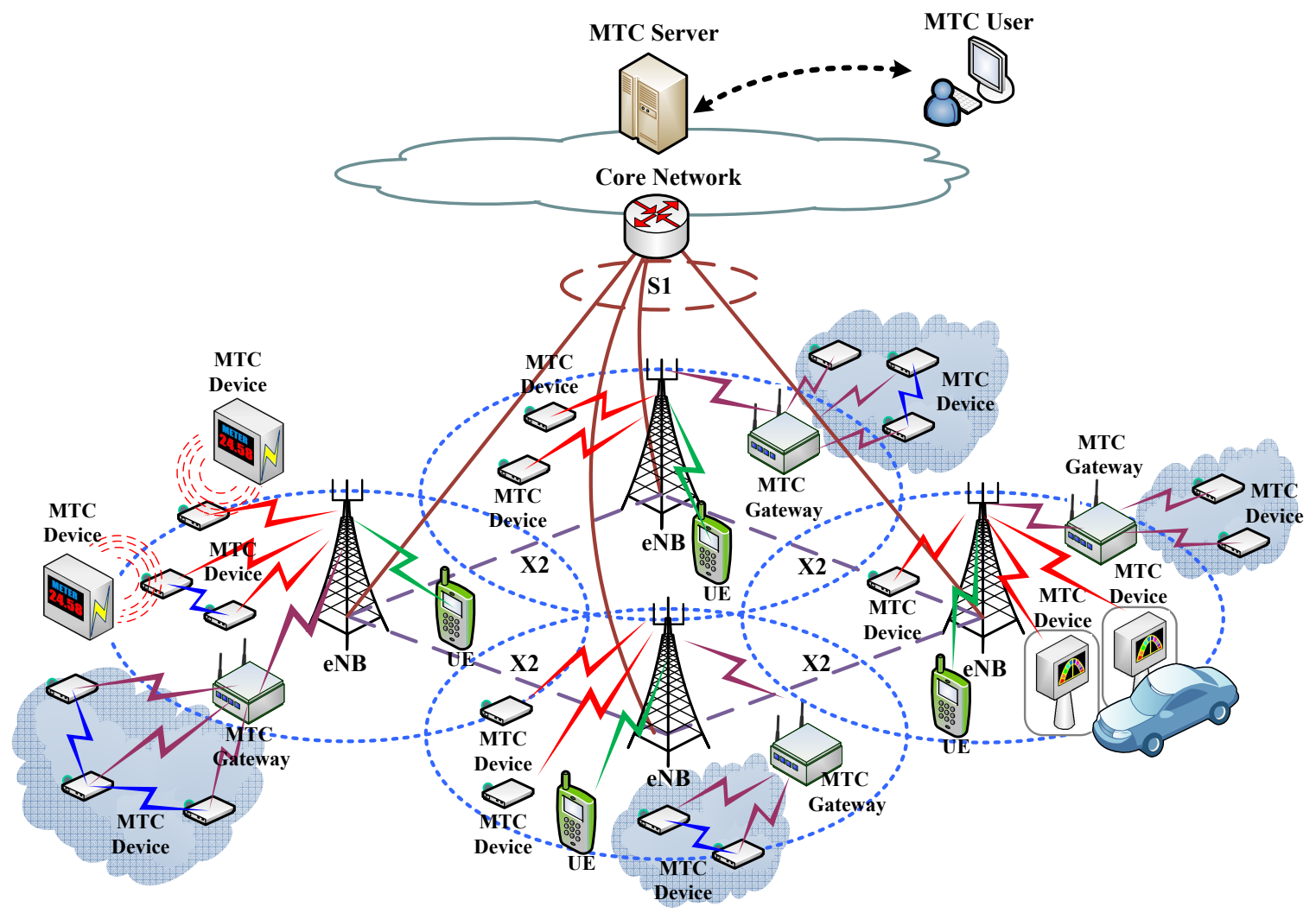

Fig. 2. Architectural enhancements to LTE-Advanced cellular networks with M2M communications. 


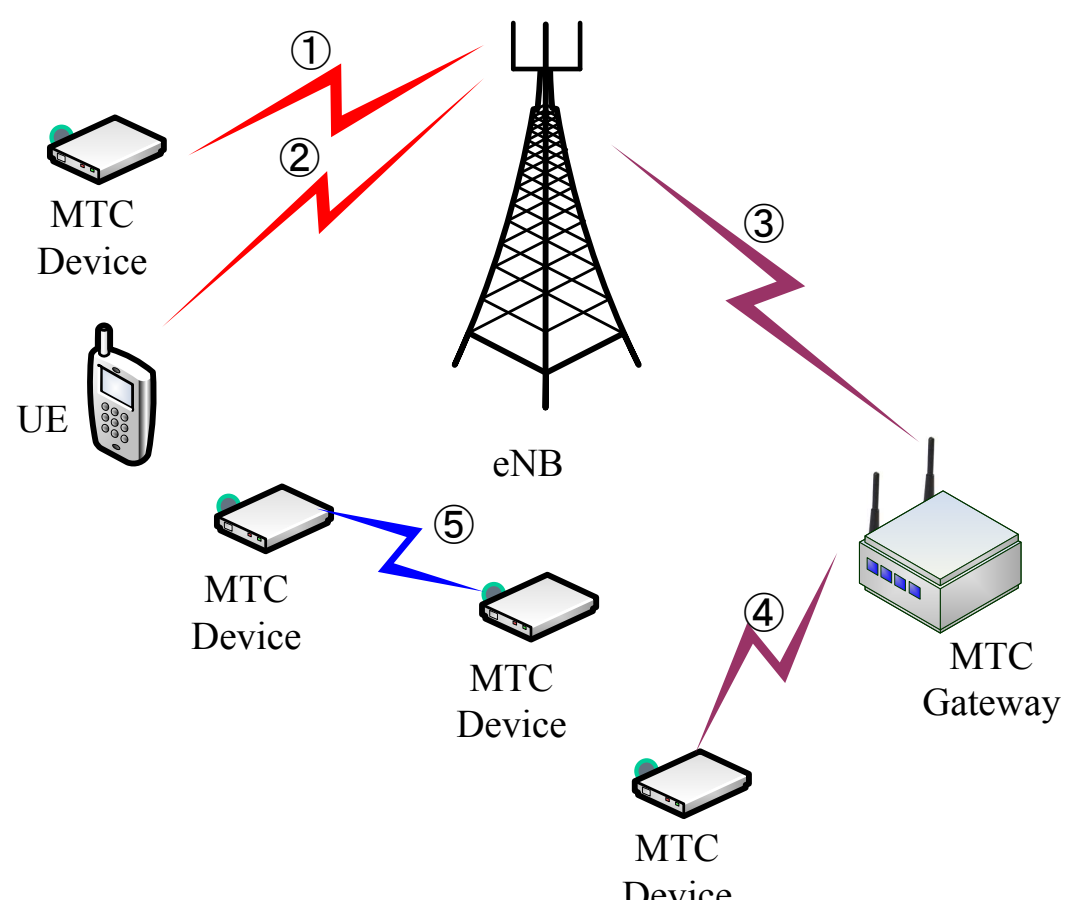

(a) Transmission links.

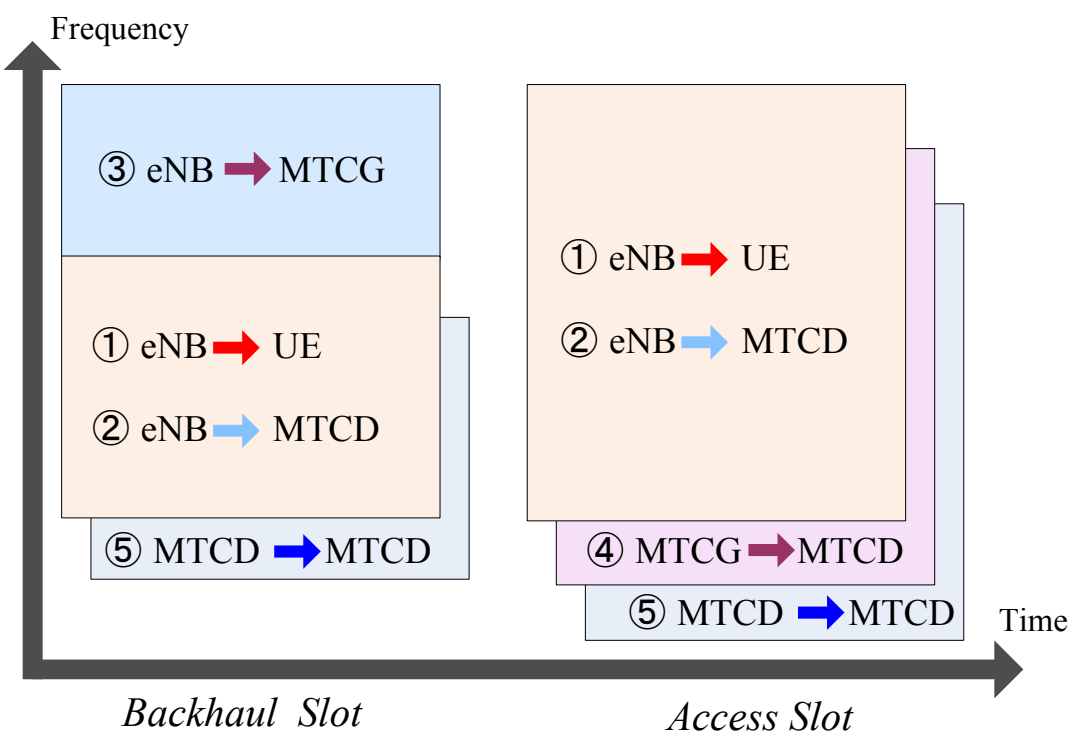

(b) Example of radio resource partition.

Fig. 3. Illustration of radio resource partition in LTE-Advanced networks with M2M communication. 


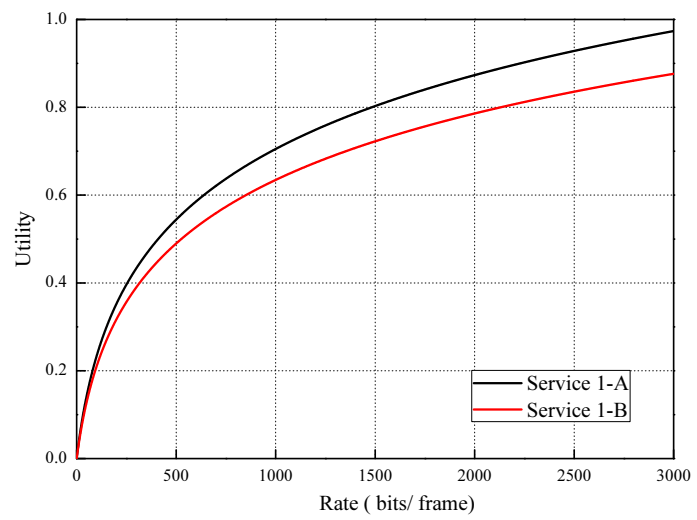

(a) Class 1 .

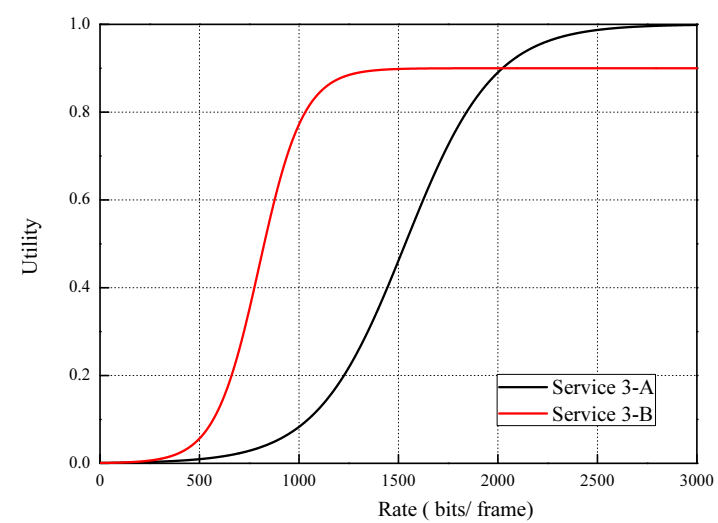

(c) Class 3 .

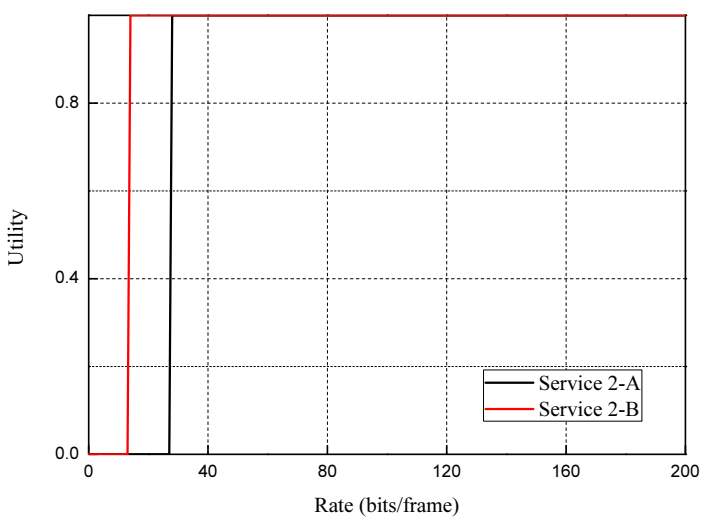

(b) Class 2 .

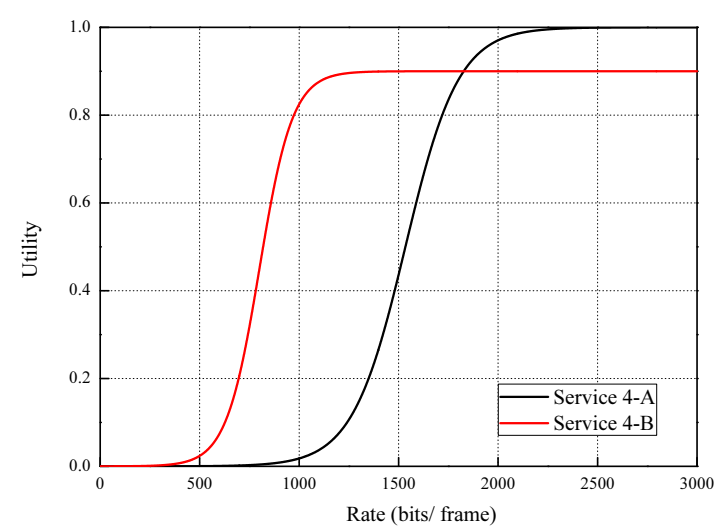

(d) Class 4 .

Fig. 4. Example utility functions for four different classes of applications. 


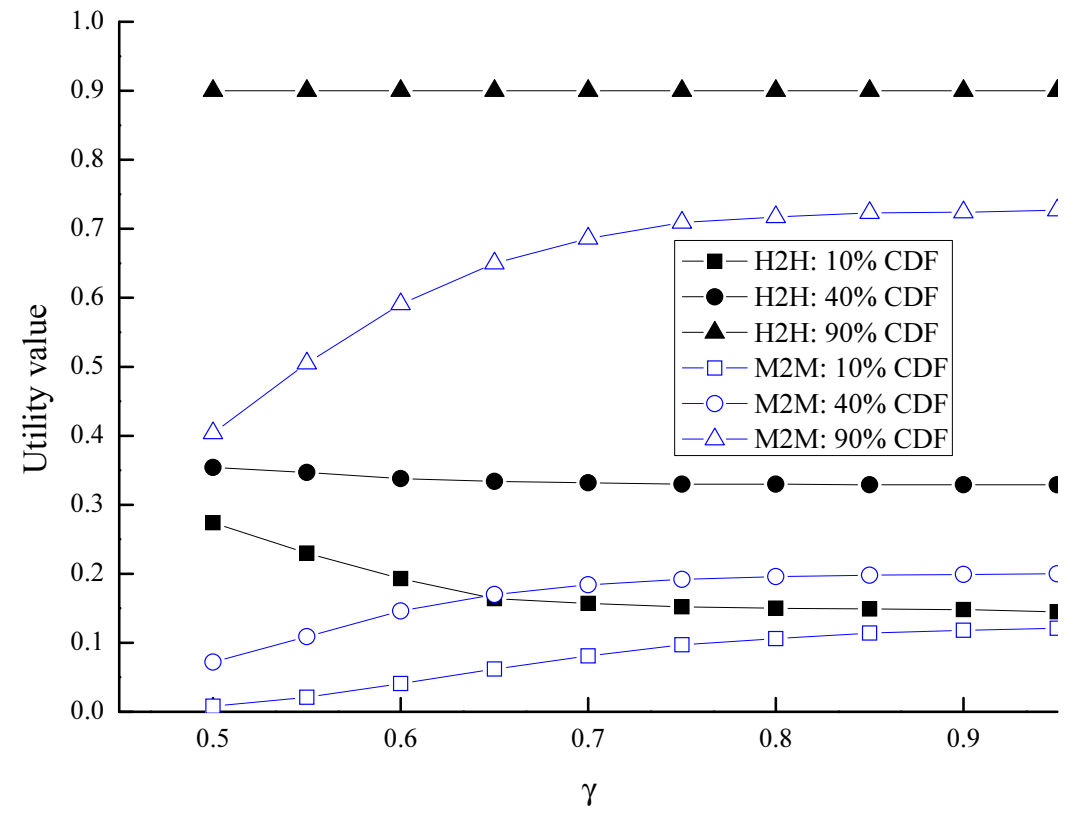

(a) Effects of unified weighting factor.

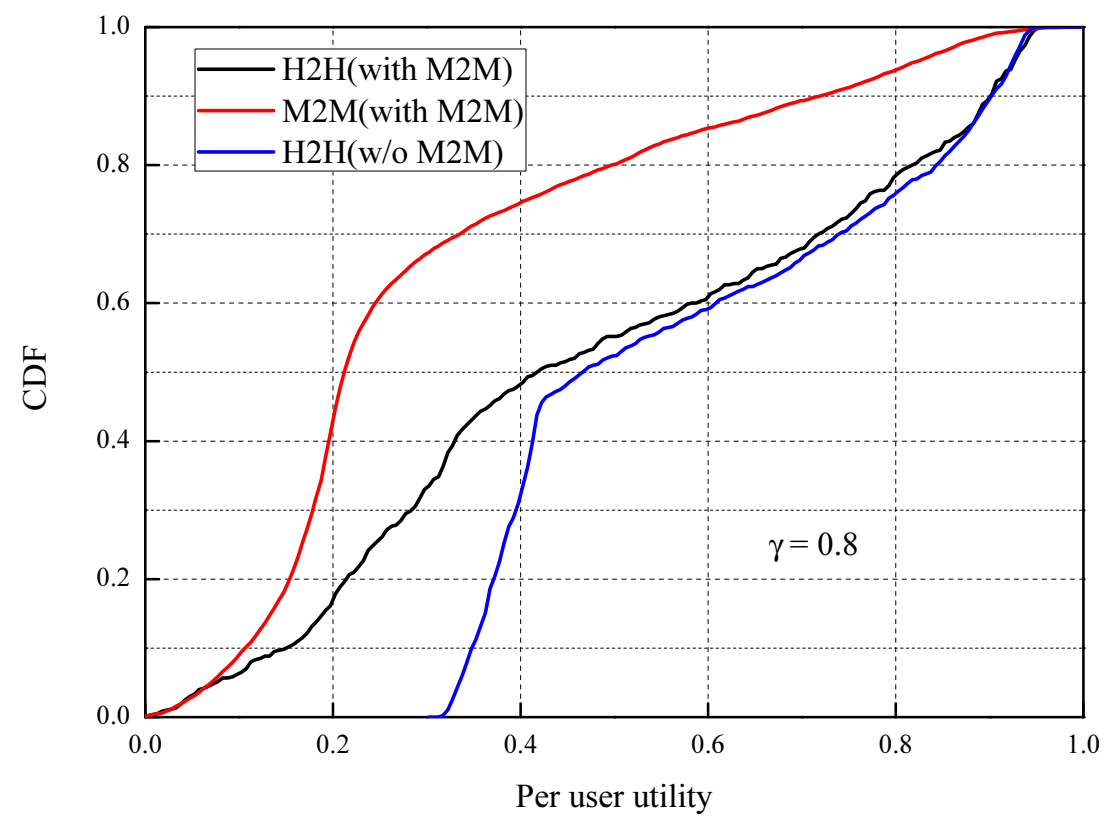

(b) CDF performances.

Fig. 5. Performance comparison in LTE-Advanced networks with/without M2M communication. 


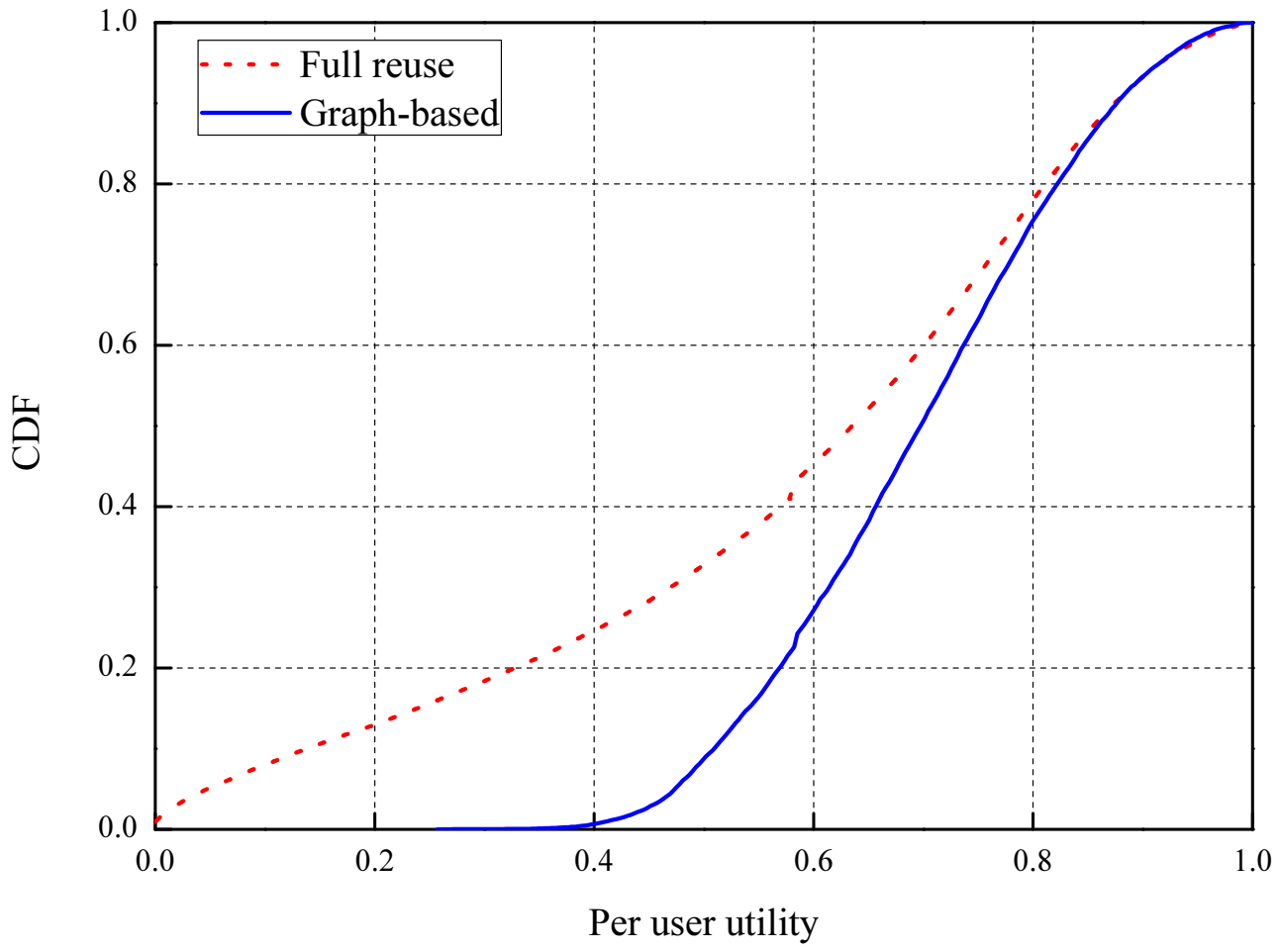

Fig. 6. Performance comparison between the full reuse and graph-based channel allocation schemes for MTCDs with peer-to-peer transmission. 